\section{IS AUTOMATED GENOMIC REPORTING READY FOR PRIME TIME?}

Leslie Burnett ${ }^{1,2,3}$

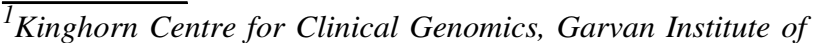
Medical Research, Darlinghurst, Sydney, NSW, Australia; ${ }^{2} S t$ Vincent's Clinical School, UNSW Sydney, Darlinghurst, Sydney, NSW, Australia; and ${ }^{3}$ Sydney Medical School, University of Sydney, Sydney, NSW, Australia

The process of reducing the large genomic datasets obtained from sequencing a genome, to a smaller and more manageable filtered set of variants for analysis, is already usually an automated pipelined process. Similarly, many laboratory and bioinformatic workflows are already amenable to basic laboratory automation.

To explore taking genomic reporting to the next stage, it is possible to utilise more powerful bioinformatic tools. These can include linear programming, large-data science, machine learning and deep learning/artificial intelligence. While some of these techniques have sophisticated mathematical underpinnings, once developed, they are surprisingly easy to apply and use.

Examples will be given of the application of these tools to clinical problems. Their diagnostic performance already exceeds the capabilities of human experts, and their speed similarly outperforms humans. Further applications include real-time point-of-care genomic diagnosis, and automated background reanalysis of unsolved cases.

\section{MACKENZIE'S MISSION}

Edwin P. Kirk $^{1,2,3}$

${ }^{1}$ Randwick Genomics Laboratory, New South Wales Health Pathology, Randwick, NSW, Australia; ${ }^{2}$ Centre for Clinical Genetics, Sydney Children's Hospital, Randwick, NSW, Australia; and ${ }^{3}$ School of Women's and Children's Health, UNSW, Randwick, NSW, Australia

Mackenzie's Mission, the Australian Reproductive Carrier Screening Project, is a research project funded by the Medical Research Future Fund that will screen 10,000 couples for 1300 genes associated with $>700$ genes. The project will study uptake, proportion of couples found to be at high risk, reproductive decisions made by those couples and psychosocial, health economic, ethics and implementation research related to carrier screening. This presentation will describe the origins and structure of the project, with a focus on the process of selecting the genes included in the screen and lessons learned from the first three months of screening.

\section{LYFE LANGUAGES AND RED FLAGS IN THE RED SAND}

Gareth Baynam $^{1,2,3,4,5,6,7,8,9}$, Yarlalu Thomas ${ }^{2}$

${ }^{1}$ Western Australian Register of Developmental Anomalies, WA, Australia; ${ }^{2}$ Genetic Services of Western Australia, WA, Australia; ${ }^{3}$ Office of Population Health Genomics, WA Health, WA, Australia; ${ }^{4}$ Faculty of Health and Medical Sciences, Division of Paediatrics, UWA School of Medicine, WA, Australia; ${ }^{5}$ University of Notre Dame, WA, Australia; ${ }^{6}$ Institute for Immunology and Infectious Diseases, Murdoch University, WA, Australia; ${ }^{7}$ School of Mines, Science and Engineering, Curtin University, WA, Australia; ${ }^{8}$ School of Population and Global Health, University of Melbourne, Vic, Australia; and ${ }^{9}$ Telethon Kids Institute, WA, Australia

Many yesterdays ago, you were created from the DNA stories you share with your ancestors. From your first day, you communicated with your mother through your face. Today, we are writing in a language that you use to share your stories, your hopes and your dreams. These are your life's languages. The life languages of our DNA, faces and the written word are universal and fundamental to health and well-being.

Gareth and Yarlalu will discuss new approaches that integrate life languages and blend ancient wisdom, old knowledge and technological innovation to generate new knowledge. Approaches that are scalable, support applications for remote diagnostics and integrate Indigenous languages for equitable access and culturally safe care. We will also discuss red flags for rare diseases and their implementation from the red sands of the Western Australian Desert to the blue waters that flow through the capital city of Western Australia, Perth, and through national and international Indigenous genomics and rare diseases networks. 